\title{
Iponatremia e fratture: risultati dello studio MrOS
}

\author{
Alessandro Peri ${ }^{1}$
}

Pubblicato online: 12 gennaio 2016

(C) Springer International Publishing AG 2016

Commento a:

Hyponatremia and fractures: findings from the MrOS study.

S.A. Jamal, S. Arampatzis, S.L. Harrison, R.C. Bucur, K. Ensrud, E.S. Orwoll, D.C. Bauer.

J Bone Miner Res (2015) 30(6):970-975

Recenti evidenze sperimentali hanno documentato l'associazione tra iponatremia, alterata qualità dell'osso e aumentato rischio di frattura. In questo lavoro è stata valutata la relazione tra iponatremia e fratture utilizzando dati provenienti dallo studio Osteoporotic Fractures in Men (MrOS). In questo studio sono stati arruolati 5122 uomini di età $\geq 65$ anni residenti in comunità e provenienti da 6 diversi centri negli USA. Dall'analisi sono stati esclusi gli uomini in terapia con bisfosfonati, quelli senza una dettagliata storia farmacologica, quelli senza determinazione della natremia. La natremia era valutata all'ingresso nello studio. I soggetti arruolati venivano seguiti per quanto riguarda la comparsa di fratture per un periodo di tempo prolungato (fino a 9 anni). Erano considerate le fratture vertebrali morfometriche (incidenti e prevalenti) e le fratture non vertebrali, incluse quelle del bacino. Per analizzare l'associazione tra i valori di natremia ( $<135 \mathrm{mmol} / \mathrm{L}$ vs $\geq 135 \mathrm{mmol} / \mathrm{L}$ ) e il rischio di frattura non vertebrale, inclusa quella di bacino, veniva utilizzato il Cox proportional hazard model e i risultati erano presentati come hazard ratio (HR), con un intervallo di confidenza (CI) del 95\%. L'associazione tra fratture morfometriche vertebrali e i valori di natremia era valutata utilizzando modelli di regressione logistica, presentati come odds ratio (OD), con un CI del 95\%.

$\triangle$ A. Peri

a.peri@dfc.unifi.it

1 Dipartimento di Scienze Biomediche Sperimentali e Cliniche "Mario Serio", Università di Firenze, Firenze, Italia
L'iponatremia era riscontrata in 64 uomini, corrispondenti all'1,2\% dell'intera coorte. Dopo aggiustamento per età, BMI, tipo di centro, e altre covariate, veniva osservato che i soggetti con natremia $<135 \mathrm{mmol} / \mathrm{L}$ avevano un rischio di frattura del bacino aumentato $(\mathrm{HR}=3,04 ; 95 \% \mathrm{CI}=$ $1,37-6,75)$ rispetto a quelli normonatremici, così come un aumentato rischio di fratture morfometriche prevalenti (OR $=2,46$; CI $0,5 \%=1,22-4,95)$ e di fratture morfometriche incidenti (OR $=3,53$; 95\% CI $=1,35-9,19)$, ma non di altre fratture non spinali $(\mathrm{HR}=1,44 ; 95 \% \mathrm{CI}=0,85-2,44)$. L'aggiustamento per la densità minerale ossea (BMD) non influenzava i risultati.

Nell'insieme, questi dati mostrano che l'iponatremia si associa a un marcato aumento del rischio di frattura sia a livello del bacino che a livello vertebrale, indipendentemente dalla BMD. Gli autori concludono che ulteriori studi sono necessari per determinare con quali modalità l'iponatremia causi fratture e se la correzione dell'iponatremia sia capace di ridurne il rischio.

Questa conclusione è del tutto appropriata, soprattutto alla luce del fatto che alcuni studi documentano, come in questo caso, che l'aumentato rischio di fratture osservato in soggetti iponatremici sia indipendente dalla ridotta BMD, mentre altri studi sperimentali mostrano una demineralizzazione ossea associata con l'iponatremia [1,2].

\section{Bibliografia}

1. Hoorn EJ, Rivadeneira F, van Meurs JB et al (2011) Mild hyponatremia as a risk factor for fractures: the Rotterdam Study. J Bone Miner Res 26:1822-1828

2. Verbalis JG, Barsony J, Sugimura Y et al (2010) Hyponatremiainduced osteoporosis. J Bone Miner Res 25:554-563 